\title{
Reingreso hospitalario en Medicina Interna
}

\author{
J.L. ALONSO MARTÍNEZ, B. LLORENTE DIEZ, M. ECHEGARAY AGARA, \\ M.A. URBIETA ECHEZARRETA, C. GONZÁLEZ ARENCIBIA
}

Servicio de Medicina Interna. Hospital de Navarra. Pamplona. Navarra

\author{
READMISSION IN INTERNAL MEDICINE
}

\section{RESUMEN}

Fundamento: La tasa de reingreso puede ser un índice de calidad asistencial, estando influenciada por múltiples factores(clínicos, derivados del hospital y del propio paciente). Analizamos el reingreso en un área clínica de medicina interna de un hospital de tercer nivel.

Material y métodos: Durante 11 meses de 1998 registramos, según un cuestionario estructurado, los reingresos (R) (ingreso en los últimos 5 años) de todos los pacientes ingresados en un área clínica de 8 camas, contabilizando número de ingresos, tiempo hasta el reingreso, área de procedencia (rural, urbana), médico de atención primaria (médico general, especialista en medicina de familia), modo de vida (sólo, familia, residencia, sin techo). Observamos la causa desencadenante del R y la enfermedad causante. Se clasifican como reingreso relacionado (RR) (reingreso por la misma enfermedad o complicación de la misma), multingreso (MR) (reingreso de una misma patología atendida en diferentes servicios del hospital), reingreso evitable (RE) (aquel reingreso que no cumple criterios $\mathrm{AEP}$ ), y reingreso temprano (RT)(reingreso antes de los 30 dÍas tras el alta).

Resultados: De un total de 312 pacientes (edad media 67,93 $\pm 15,5$, $64 \%$ varones, estancia media 7,75 $\pm 4,35$ días, ingresos urgente $93 \%$, tasa de mortalidad 3,52\%). Fueron R $111(35,5 \%)$, RR 83 (26\% del total y $75 \%$ de R), MR 68 (61,2 de R y 82\% de RR), RT 33 (39,7\% de RR) y 16 RE (19\% de RR). No hubo diferencias en edades, sexo ni estancia media.

Las enfermedades más frecuentes fueron la insuficiencia cardiaca y las enfermedades respiratorias crónicas. Las causas del $\mathrm{R}$ fueron empeoramiento de trastorno crónico 41 (37\%), manejo inadecuado ambulatorio $24(22 \%)$, mal diagnóstico previo $8(7 \%)$, efecto tóxico-iatrogenia 7 (6\%), nueva enfermedad $29(36 \%)$, otros $2(2 \%)$. El número de ingresos previos era de $3,22 \pm 2,25$ y el tiempo hasta el reingreso de $8,99 \pm 11,96$ meses. La tasa de mortalidad intrahospitalaria de los $\mathrm{R}$ fue de $7,2 \%$ $(\mathrm{p}<0,05)$ (intervalo de confianza al $95 \%$ de 2 a $9 \%)$. Vivir en zona urbana $(\mathrm{p}<0,05)$ y tener un médico de familia como médico de atención primaria $(\mathrm{p}<0,01)$ fueron factores aceleradores del $\mathrm{R}$.

Los pacientes RR presentaban mayor número de ingresos previos $(3,53 \pm 2,23)(\mathrm{p}<0,001)$ y reingresaron antes $(8,03 \pm 11,85)(\mathrm{p}<0,01)$ con una tendencia mayor al reingreso en las mujeres $(\mathrm{p}=0,052)$. No consultaron con atención primaria $57 \%$ de los pacientes $(\mathrm{p}<0,05)$ (intervalo de confianza a $95 \% 3$ a 39\%). la consulta con atención primaria supuso un retardo en el reingreso de 5 meses $(\mathrm{p}<0,01)$ y el manejo ambulatorio inadecuado se asoció a aceleración en el reingreso $(p<0,05)$.

Los pacientes MR tenían mayor número de ingresos previos $(p<0,001)$ y eran factores favorecedores la toxicidad-iatrogenia $(p<0,05)$, manejo ambulatorio inadecuado $\mathrm{p}<0,001$ ) y empeoramiento del trastorno crónico $(\mathrm{p}<0,001)$.

Los pacientes con RT presentaron mayor mortalidad intrahospitalaria $(\mathrm{p}<0,05)$.

\section{ABSTRACT}

Background: The readmission rate could be a valuable tool as mea surement of hospital quality. Readmissions are due to several factors: clinical, hospital related and patient related. We analyze readmission to internal medicine in a hospital of third level.

Material and methods: During 11 months in 1988 we counted all readmissions $(R)$ defined as every previous admission occurred in a span of five years into an area of internal medicine composed by 8 beds. We counted number of readmssions, time from the last readmission, living area (city vs country), sort of primary care physician (GP vs family care specialist), living way (single, with family, institution, homeless). Preci pitating factors were observed as well as diseases causing it. $R$ were classified as $R$ related $(R R)$ when readmission was provoked by the same pathoiogical condition or a complication. Multi-readmission (MR), those $R$ caused by the same disease process and treated in different areas and ervices of the hospital. Avoidable $R(A R)$, those $R$ which did not fillfil $A E P$ criteria. Early readmission (ER) those $R$ occurring before 30 days after last discharge.

Results: Three hundred and eleven patients (mean age 67.93 (SD 15.51), males 64\%, mean length of stay 7.75 (SD 4.35), 93\% admitted from emergency yard, mortality rate $3.5 \%)$ were included. $R$ were 111 (35.5\%), RR 83 (26 and 75\% of RR), MR 68 (82\% of RR), ER 33 (39.7\% of $R R)$ and $A R 16(19,2 \%$ of $R R)$ patients.

The most frequent diseases were heart failure and chronic respira tory diseases. Main causes of $R$ were worsening of chronic disease 41 (37\%), non-appropriale ambulatory management 24 (22\%) erroneous diagnosis $8(7 \%)$, iatrogenic effec 7 (6\%), new disease $29(26 \%)$ and others $2(2 \%)$. Mortality rate in R patients was $7.2 \%$ (confidence interval 95\% 2 to 9\%). Number of readmissions were 3.22 (SD 2.25) and time to readmission 8.99 (SD 11.96) months. Living in city $(p<0.05)$ and to be cared by family physician $(p<0.01)$ both were factors accelerating read mission.

Patients with RR had a higher number of readmissions (3.55 SD $2.23 p<0.001)$ and they occurred sooner (8.03 SD 11.85) $(p<0.01)$. There was a trend to higher readmission rate in female ( $p 0.052)$. Fifty seven percent of RR patients did not have consultation with primary care physician $(p<0.05)$ (confidence interval 95\% 3 to 39\%). Consulta tion with primary care yielded a delay in readmission of 5 months $(p<0.01)$.

Patients with MR had an increased number of readmissions $(p<0.01)$. Associated factors were iatrogenic effect $(p<0.05)$, non-appro priate ambulatory management $(p<0.001)$ and worsening chronic disea se $(p<0.001)$.

Patients with ER were readmitted 0.45 (SD 0.30) months after the last discharge and they had a higher mortality rate $(p<0.05)$.

Trabajo aceptado: 15 de Noviembre de 2000 
Los pacientes con RE tuvieron una estancia media más corta $(\mathrm{p}<0,05)$, tendencia a mayor numero de ingresos $(\mathrm{p}=0,06)$ y éstos a ocurrir antes $(\mathrm{p}=0,08)$ con una mortalidad nula $(\mathrm{p}<0,01)$.

En regresión logística los factores de riesgo asociados a RR fueron ser MR, RT, tener un RE y como causa de R iatrogenia, manejo ambulatorio inadecuado, empeoramiento de enfermedad crónica o mal diagnostico previo.

Conclusiones: La tasa de reingreso verdadero es de $26 \%$, siendo la insuficiencia cardiaca y las enfermedades respiratorias crónicas las principales enfermedades, con tendencia a ocurrir más en mujeres. Hasta $39 \%$ de los reingresos pueden ser prevenibles. Existe un fenómeno de facilitación en el número y en el tiempo de reingreso con bajo índice de consulta con atención primaria. Los factores de riesgo asociados a reingreso verdadero son el multingreso, el reingreso temprano, el tener un reingreso evitable y tener como causa de reingreso iatrogenia, manejo ambulatorio inadecuado, empeoramiento de enfermedad crónica y diagnóstico previo erróneo.

PALABRAS CLAVE: Reingreso hospitalario. Servicios de Medicina Interna
Patients with AR had a mean length of stay shorter ( $p<0.05)$, a trend to higher readmission rate $(p=0.06)$ and sooner $(p=0.08)$ with a null mortality rate $(p<0.01)$.

As risk factors for $R R$ in logistic regression were identified $M R, A R$ $E R$ and causes of readmission consisting in worsening of chronic disea se, non-appropriate ambulatory management, erroneous diagnosis and iatrogenic effect.

Conclusions: Our readmission rate is $26 \%$, chronic respiratory dise ases and heart failure being the main diseases. Over $39 \%$ of causes of readmission could be preventable and there is a facilitation phenomenon in number and time to readmission caused by previous readmissions. Risk factors for readmission in internal medicine are multi-readmission, early and avoidable readmission and as specific causes worsening of chronic disease, non-appropriate ambulatory management, erroneous diagnosis and iatrogenic effect.

KEY WORDS: Readmission. Internal Medicine.

\section{INTRODUCCIÓN}

El reingreso hospitalario se ha estudiado como índice de calidad asistencial desde al menos 1965 (1), habiendo sido considerado como uno de los estándares de calidad hospitalaria (2-5), aunque se ha señalado que no debe ser considerado como un resultado final, en el sentido de que evitar el reingreso no sería un objetivo directo de los cuidados hospitalarios (2).

En los últimos años se duda que la tasa bruta de reingreso sirva como índice de calidad $(6,10)$, considerándose en algunos trabajos como debido a causas no modificables, aunque una tasa alta de reingreso en determinadas patologías podría identificar problemas en la asistencia prestada $(5,7)$. Además pueden existir diferencias entre el reingreso programado y aquellos no programados, por lo que estos últimos son los que debieran ser utilizados como índice de calidad (8). Así, se ha señalado la utilidad de dividir el reingreso según sea debido a complicaciones del ingreso previo, recurrencia de la enfermedad, adherencia al tratamiento planeado o enfermedad nueva (9).

Otros autores sí consideran que el reingreso hospitalario puede ser indicativo de mala calidad de los cuidados hospitalarios, particularmente en algunas enfermedades con alta tasa de recurrencia y cronicidad (11-13), aumentando el riesgo de reingreso hasta en $55 \%$ cuando los cuidados ofrecidos no han sido de suficiente calidad $(14,15)$, siendo la variabilidad clínica en el manejo de un mismo proceso clínico un factor que se asocia con un incremento en la tasa de reingreso $(16,17)$.

En España, la tasa de reingreso hospitalario comunicada es muy variable $(48,49)$, existiendo variabilidad en las series presentadas en cuanto a la patología causante, la consideración de reingreso temprano, la terminología empleada (readmisión frente a reingreso) y datos contradictorios sobre su utilidad como índice de calidad asistencial. A pesar de todos estos obstáculos, el conocimiento del reingreso en un área determinada de trabajo, puede servir como indicador de calidad local.

\section{MATERIAL Y MÉTODOS}

Durante 11 meses de 1998 (no se evaluó julio), mediante un cuestionario estructurado recogido a partir de la historia clínica, que consta de fecha de ingresos previos, servicio o unidad de ingreso, causa y diagnostico principal de alta, se han registrado todos los pacientes ingresados en un área clínica de medicina interna de 8 camas. Se constataron todos los eventos que causaron ingreso hospitalario en este $u$ otro centro durante los 5 años previos. Asimismo se recogieron área de vivienda habitual (urbana o rural), tipo de médico de asistencia primaria (médico de familia, médico general), modo de vida (con familia, familia itinerante, solo, residencia de ancianos, centro de crónicos).

Consideramos tres tipo de reingreso: Reingreso Total (R), aquellos pacientes que por cualquier motivo hubieran estado ingresados en algún hospital durante los 5 años previos. Rein greso Relacionado (RR) o verdadero reingreso, aquellos pacientes que reingresan por la misma patología que causó el o los ingresos previos o por complicaciones de la misma. Mul tingreso (MR), aquellos pacientes que reingresan en diferentes servicios o secciones del hospital para tratar la misma patología (por ejemplo un paciente con disnea puede ser ingresado en nuestro centro en medicina interna, cardiología, neumología, y geriatría, o un paciente con dolor abdominal puede ser ingresado en medicina interna, cirugía, digestivo o geriatría).

Se constataron la enfermedad principal causante del reingreso y la comorbilidad, lo adecuado del ingreso mediante aplicación del Protocolo de Adecuación del Ingreso Hospitalario, $\operatorname{AEP}(46,47)$ (Reingreso Evitable RE, aquel que no cumplía criterios AEP) la edad del paciente, el número de ingresos previos, el ingreso programado o no programado y la tasa de mortalidad. Se midió el tiempo de ingreso desde la última fecha de alta y se clasificó como Reingreso Temprano (RT) aquel que ocurre en los 30 días siguientes a la fecha de la última alta. 
Se recogió de forma específica el motivo del R: descompensación de la enfermedad crónica, manejo ambulatorio inadecuado, toxicidad-iatrogenia, diagnóstico previo erróneo, y otros motivos, así como si antes del $\mathrm{R}$ el paciente había consultado con el médico de atención primaria o no.

El hospital es un centro terciario con todas las especialidades médicas desarrolladas y funcionando como servicios o secciones autónomas.

Análisis estadístico. Para variables continuas se utiliza la media y la desviación estándar como medida de dispersión. La comprobación de distribución normal de variables continuas se realizó con la prueba de Kolmogorov Smirnov para comparación de medias se utilizó la prueba de la t de Student cuando tenían distribución normal y la prueba de la u de Mann Whitney para variables sin distribución normal. Para comparación de variables discretas su utilizó la prueba de comparación de proporciones, la de ji al cuadrado y la prueba exacta de Fisher. Utilizamos ANOVA para calcular el efecto de variables continuas con distribución normal con respecto a discretas y la prueba de Kruskal Wallis cuando no tenían distribución normal.

Con regresión logística construimos un modelo paso a paso de identificación de múltiples factores influenciando el RR.

Se consideró significación los valores de $\mathrm{p}<0,05$ y tendencia los valores de $\mathrm{p}<0,1)$.

\section{RESULTADOS}

\section{TOTAL DE PACIENTES}

Un total de 312 pacientes ingresaron durante el periodo de estudio con una edad media de 67,93 $\pm 15,51$ años, hombres $168(64 \%)$, mujeres 144 (46\%), que cursaron una estancia media de 7,75 $\pm 4,35$ días. Fueron ingreso desde urgencias 282 (93\%), de UCI $2(0,5 \%)$, de consultas externas 14 (4,5\%) y de otros servicios $6(2 \%)$. Fallecieron 11 pacientes durante el ingreso (índice de mortalidad 3,52\%).

Ciento once fueron R (35,5\%); 83 fueron RR $(26,6 \%$ del total y $75 \%$ de los R); 68 pacientes fueron MR $(21,79 \%$ del total, $61,2 \%$ de los R y $81,9 \%$ de los RR); RT fueron 33 pacientes (10,5\% del total, $29,7 \%$ de $\mathrm{R}$ y $39,7 \%$ de RR), 16 pacientes fueron RE (19,2\% de RR) (Fig. 1).

\section{REINGRESO TOTAL}

Reingreso total (R) fueron 111 pacientes $(35,5 \%)$, hombres $58(52 \%)$ y mujeres $53(48 \%)$ con una edad media de $70,18 \pm 12,20$ años, que cursaron una estancia media de $8,03 \pm 4,81$ días. Procedían de área rural $52(47 \%)$ de los pacientes, y de área urbana 59 (53\%). El médico de atención primaria correspondiente era médico de familia en $57(51,4 \%)$ y médico general en $54(48,6 \%)$ pacientes. Noventa y cuatro pacientes $(85 \%)$ vivían en ambiente familiar. El ambiente familiar (modo de vida) no tuvo influencia en los R.

Fueron ingresos urgentes no programados todos los pacientes: 108 (97\%) desde urgencias y $3(3 \%)$ ingresos desde consulta externa. Se dieron de alta $103(93 \%)$ y fallecieron 8 (7\%). La causa más frecuente de $\mathrm{R}$ fue el empeoramiento de la enfermedad crónica, pero hasta el $39 \%$ de los $\mathrm{R}$ son debidos a causas modificables (manejo ambulatorio inadecuado, iatrogenia, mal diagnóstico previo).

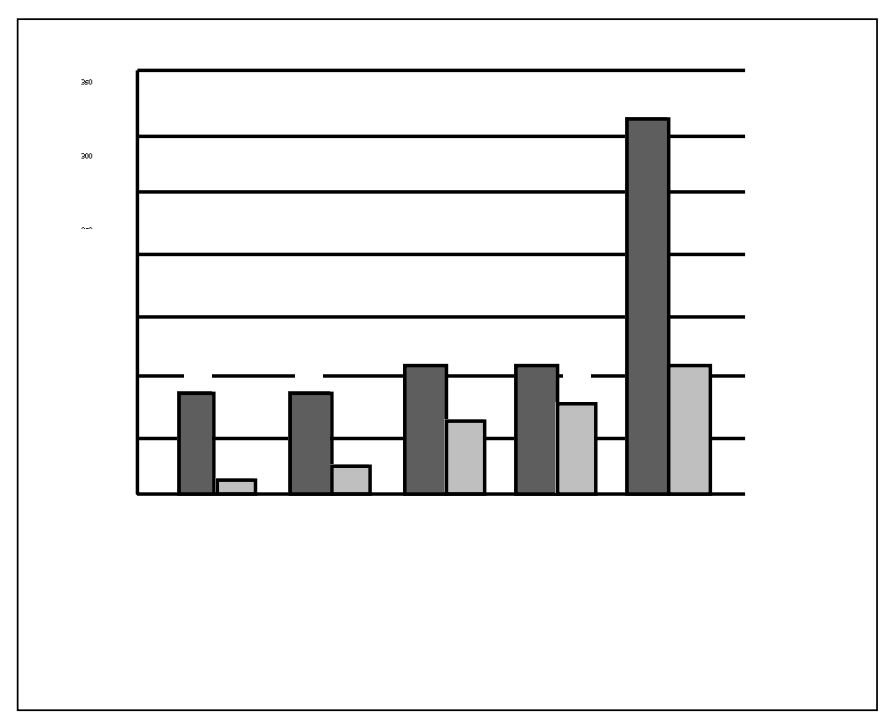

Fig. 1.

No hubo diferencias de edad, estancia media ni proporción de sexos pero la proporción de muertes fue mayor en el grupo $\mathrm{R}(\mathrm{p}<0,05)$ (intervalo de confianza $95 \%$ de 2 a $9 \%$ ) respecto al total de pacientes.

Los pacientes $\mathrm{R}$ que fallecieron tuvieron una estancia media más corta $(5 \pm 4)$ que los que sobrevivieron $8,28 \pm 4,80$ $(\mathrm{p}<0,05)$.

El tiempo transcurrido desde el último ingreso fue de $8,99 \pm 11,96$ meses. El número de ingresos previos era de $3,22 \pm 2,25$.

En la figura 2 se muestra la procedencia de ingresos previos por servicios y unidades de los pacientes $\mathrm{R}$ y de los pacientes RR.

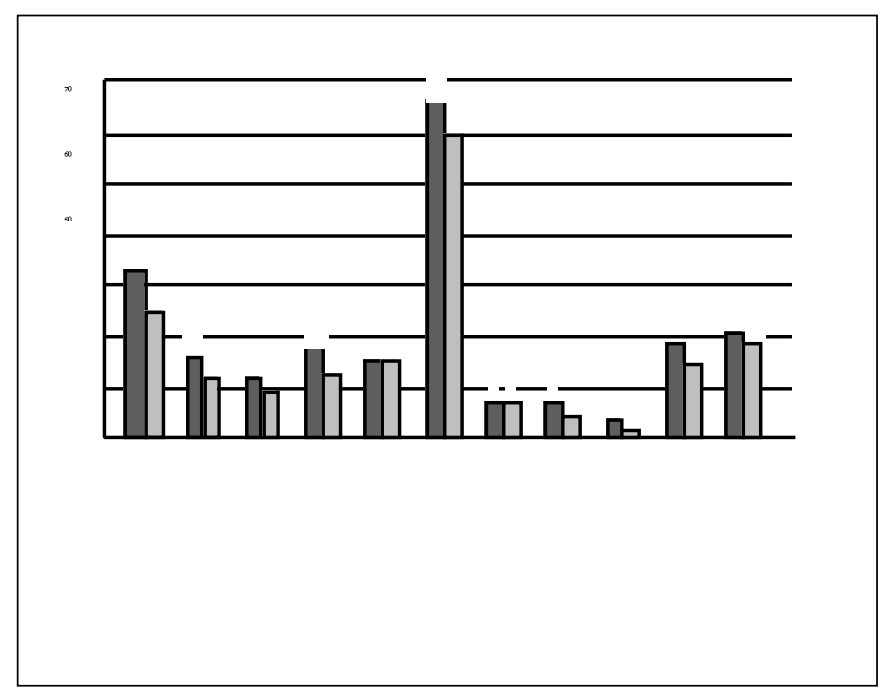

Fig. 2.

Cuarenta pacientes $(35 \%)$ eran controlados en consultas externas por algún servicio, mientras que 71 (64\%) no lo eran. Este hecho no influenció la estancia media del R. Eran R de este hospital $102(92 \%)$, mientras que 9 (8\%) lo eran de otros hospitales. En las figuras 3 y 4 se muestran las patologías causantes del R y la comorbilidad acompañante respectivamente. 


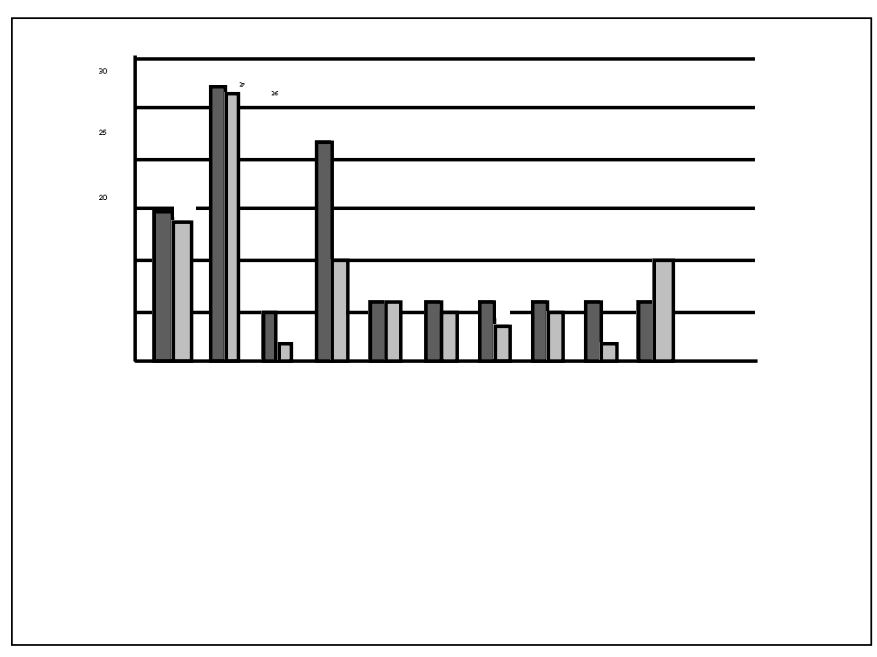

Fig. 3.

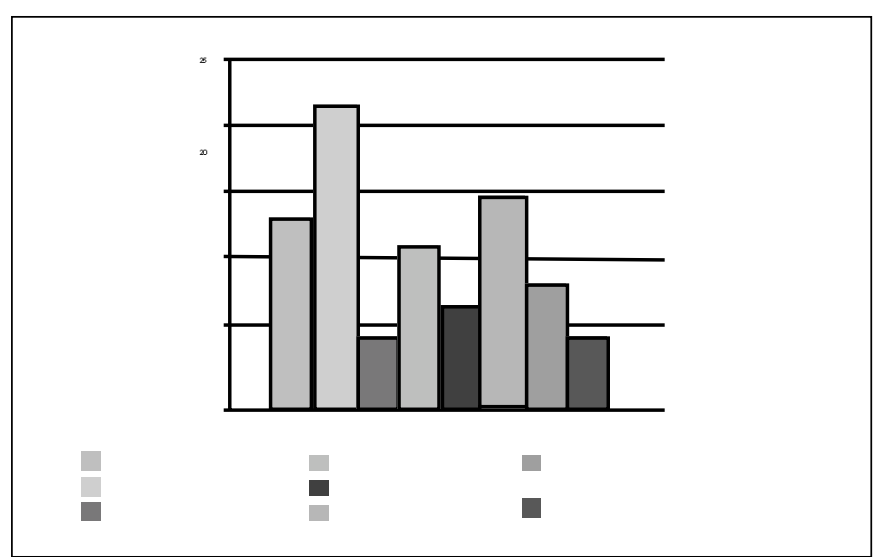

Fig. 4.

Las causas del R son mostradas en la tabla I, y en la tabla II se muestran los tiempos transcurridos desde el último ingreso.

En la tabla III se especifican los tiempos de R según diferentes circunstancias.

\section{REINGRESO RELACIONADO (RR)}

No hubo diferencias en la edad, estancia media y proporción de sexos respecto al grupo $\mathrm{R}$ ni al total de pacientes.

\section{TABLA I}

CAUSAS DEL REINGRESO

\begin{tabular}{lll}
\hline & $\mathrm{R}$ & $\mathrm{RR}$ \\
\hline $\begin{array}{l}\text { Empeoramiento del } \\
\text { trastorno crónico }\end{array}$ & $41(37 \%)$ & $41(49 \%)$ \\
$\begin{array}{l}\text { Manejo ambulatorio } \\
\text { inadecuado }\end{array}$ & $24(22 \%)$ & $24(29 \%)$ \\
Mal diagnóstico previo & $8(7 \%)$ & $8(10 \%)$ \\
Efecto tóxico-iatrogenia & $7(6 \%)$ & $6(8 \%)$ \\
Nueva patología & $29(26 \%)$ & $2(2 \%)$ \\
Otros & $2(2 \%)$ & $2(2 \%)$
\end{tabular}

TABLA II

\begin{tabular}{llll}
\multicolumn{4}{c}{ TABLA II } \\
TIEM PO HASTA EL REINGRESO \\
\hline Causa & Tiempo (meses) & p \\
\hline M anejo inadecuado & $6,66 \pm 12,66$ & 4 respecto $1<0,05$ \\
Toxicidad iatrogenia & $7,33 \pm 10,57$ & 1 respecto $2=0,06$ \\
Diagnóstico previo erróneo & $8,7 \pm 12,19$ & 1 respecto $5<0,05$ \\
Empeoramiento de & & & \\
enfermedad crónica & $9,23 \pm 11,45$ & \\
Nueva patología & $10,91 \pm 12,90$ &
\end{tabular}

TABLA III

TIEM POS HASTA EL REINGRESO

\begin{tabular}{llll}
\hline & Sí & No & p \\
\hline Relacionado con ingresos & & & \\
$\begin{array}{c}\text { previos (RR) } \\
\text { Reingreso evitable (RE) }\end{array}$ & $8,03 \pm 11,85$ & $11,81 \pm 12,04$ & $<0,01$ \\
Consulta con Atención-1a & $9,18 \pm 11,89$ & $7,82 \pm 12,68$ & $=0,08$ \\
& $10,77 \pm 12,39$ & $7,63 \pm 11,53$ & $<0,01$ \\
& Rural & Urbana & \\
Procedencia & $10,06 \pm 11,94$ & $8,03 \pm 11,99$ & $<0,05$ \\
& Familiar & General & \\
Tipo de médico & $7,39 \pm 11,19$ & $10,66 \pm 12,60$ & $<0,01$ \\
& Urbana-Familia & Urbana-General & \\
Tipo de médico y área & & & \\
de salud & $7,52 \pm 11,44$ & $13,56 \pm 17,57$ & $<0,05$
\end{tabular}

Los pacientes del grupo RR tenían un número de ingresos previos de 3,53 $\pm 2,23$ mientras que los del grupo $\mathrm{R}$ eran de $2,32 \pm 2,10(\mathrm{p}<0,001)$. El tiempo transcurrido desde el ingreso previo fue en el grupo RR de 8,03 \pm 1 1,85 meses, y en el grupo

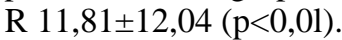

Los pacientes mayores de 70 años tuvieron una estancia media de 7,11 $\pm 3,83$ y los menores de 70 años de $8,36 \pm 4,16$ $(\mathrm{p}=0,055)$. El número de ingresos previos fue en los varones de $3,23 \pm 2,26$ y en las mujeres $3,85 \pm 2,10$ ( $p=0,052)$.

La proporción de RT fue de $36 \%$ y la de RD $11 \%$ (p 0,01) (intervalo de confianza 0,07 a 0,43 , p<0,05), y la de MR $76 \%$ mientras fue $18 \%$ en el grupo $\mathrm{R}(\mathrm{p}<0,001)$, (intervalo de confianza para $\mathrm{p}<0,05$ de 38 a $77 \%$ ).

La proporción de consulta con $\mathrm{AT}-\mathrm{l}^{\mathrm{a}}$ era de $36 \%$ mientras no consultaron $64 \%(\mathrm{p}<0,01)$ (intervalo de confianza $\mathrm{p}<0,05$ de 8 a $40 \%$ ). El tiempo transcurrido hasta el reingreso cuando consultaban con AT- $1^{\circ}$ fue de $11,42 \pm 13,70$ meses y de $6,11 \pm 10,31$ meses cuando no lo hacían $(p<0,01)$.

En la tabla IV se muestran los tiempos medios desde el último ingreso según las diferentes causas de RR.

\section{MULTINGRESO (MR)}

No hubo diferencias en la edad de los pacientes ni en la estancia media. Había una tendencia en los varones a causar MR $(68 \%)$ respecto a las mujeres $(53 \%)(\mathrm{p}=0,07)$.

El número de ingresos previos en el grupo MR fue de $3,54 \pm 2,14$ mientras que en el resto fue de 2,72 $\pm 2,35(\mathrm{p}<0,01)$. No hubo diferencias en el tiempo de reingreso, en la propor- 
TABLA IV

\section{TIEM POS DE REINGRESO EN RR}

\begin{tabular}{lll}
\hline Manejo inadecuado & $6,38 \pm 13,51$ & 1 respecto $4 \quad p<0,05$ \\
Toxicidad iatrogenia & $3,72 \pm 4,97$ & 1 respecto $3 \quad p<0,05$ \\
Diagnóstico previo erróneo & $8,7 \pm 12,194$ & \\
Empeoramiento de & & \\
$\quad$ enfermedad crónica & $9,38 \pm 11,555$ & \\
Otros & $7,27 \pm 14,59$
\end{tabular}

ción de RT y RD, en la mortalidad, en la proporción de consultas externas, RE, área de salud ni tipo de médico.

No hubo diferencias en el tiempo de reingreso según las diferentes patologías, pero sí en la proporción de causas de reingreso: era más frecuente el reingreso por toxicidad $(\mathrm{p}<0,05)$, por manejo inadecuado $(\mathrm{p}<0,001)$, y por empeoramiento de la enfermedad crónica $(\mathrm{p}<0,001)$.

\section{REINGRESO TEMPRANO (RT)}

No se demostraron diferencias entre RT y RD en edades, estancia media ni proporción de sexos. El RT presentó una mortalidad intrahospitalaria de $18 \%$, mientras que en el R tardío fue de $2(3 \%)(\mathrm{p}<0,05)$. Los RT ocurrieron $0,45 \pm 0,30$ meses tras el alta. Los pacientes con RT habían causado $3,93 \pm 2,94$ ingresos previos mientras que los pacientes con $\mathrm{R}$ tardío eran de $2,92 \pm 1,82(\mathrm{p}=0,08)$.

RT se asoció a $R R(p<0,05)$, a $R E(p<0,05)$ a no consulta con Atención Primaria $(\mathrm{p}<0,001)$ y a área de salud urbana $(\mathrm{p}<0,05)$.

\section{REINGRESO EVITABLE (RE)}

La estancia media de los RE fue 5,92 $\pm 2,61$ días, mientras que la de los $\mathrm{R}$ adecuados fue 8,35 $\pm 4,99$ días $(\mathrm{p}<0,05)$. El número de ingresos previos en RE fue de $3,81 \pm 2,22$ días, y en los $\mathrm{R}$ adecuados fue de $3,12 \pm 2,25$ ( $\mathrm{p}=0,06$ ), el tiempo hasta el reingreso fue en $R E$ de 7,82 $\pm 12,68$ meses, mientras que en $R$ adecuado fue de $9,18 \pm 11,89(\mathrm{p}=0,08)$. La proporción de $\mathrm{RT}$ fue de $27 \%$ mientras que fue $\mathrm{R}$ tardío en $9 \%(\mathrm{p}<0,05)$ (intervalo de confianza $95 \%$ de 0,3 a $36 \%$ ).

Ningún paciente de los RE falleció en el hospital respecto a los $\mathrm{R}$ adecuados $(\mathrm{p}<0,01)$. El manejo ambulatorio inadecuado se asoció a $R E(p<0,001)$.

\section{REGRESIÓN LOGÍSTICA}

En un modelo paso a paso, siendo la variable independiente RR, encontramos que los mejores predictores de reingreso eran haber sido multingreso, tener un reingreso evitable, ser reingreso temprano y como causa de reingreso consideradas de forma global haber presentado iatrogenia, manejo inadecuado ambulatorio, diagnóstico previo erróneo o empeoramiento de la enfermedad crónica. En la tabla V se expresan los odds ratio para los diferentes factores.

\begin{tabular}{lllll} 
& \multicolumn{4}{c}{ TABLA V } \\
& $\mathrm{b}$ & $\mathrm{p}$ & $\mathrm{OR}(\mathrm{IC} 95 \%)$ & ORst \\
\hline Independiente & $-8,72$ & 0,0001 & & \\
Multingreso & 3,29 & 0,0005 & $26,84(4,44-162,35)$ & 5,01 \\
Causa de reingreso & 1,12 & 0,0003 & $3,06(1,85-5,09)$ & 7,17 \\
Reingreso temprano & 3,03 & 0,006 & $20,72(2,39-179,17)$ & 4,03 \\
Reingreso evitable & 2,07 & 0,093 & $7,96(0,72-88,12)$ & 2,08 \\
\hline
\end{tabular}

b: coeficiente de regresión; OR (IC 95\%) odds ratio e intervalo de confianza al 95\% ; O Rst: razón de predominio estandarizado.

\section{DISCUSIÓN}

El reingreso hospitalario se contempla como índice de calidad asistencial hospitalaria desde los años 60 (1), habiéndose defendido como uno de los estándares de calidad (25). No obstante, en parte relacionado con la diferente terminología empleada, también se ha dudado de su utilidad como índice general $(6,10)$, aunque en determinados procesos clínicos (p. ej., insuficiencia cardiaca, asma bronquial) la tasa de reingreso puede identificar problemas en la calidad asistencial $(5,7,11-13)$.

Múltiples causas han demostrado su influencia en la tasa de reingreso: complicaciones del ingreso previo, recurrencia de la enfermedad, mal cumplimiento terapéutico o aparición de una enfermedad nueva $(7,9)$. Un hecho que se considera importante a la hora de valorar la tasa de reingreso es el diferenciar entre reingreso programado y no programado, inclinándose la literatura a tratar solo de estos últimos en la valoración de la tasa de reingreso $(6,8)$.

Otros muchos factores se han estudiado en relación con la tasa de reingreso, unos dependientes del paciente y su entorno social: edad, grado de dependencia, nivel económico, estado de salud autopercibido (19-24) tipo de seguro médico (22), educación del paciente (27) y de la familia (28) respecto al problema de salud específico. Otros factores son dependientes del tipo de enfermedad $(8,21,24,25)$, siendo en general la tasa de reingreso más baja cuando la enfermedad es de tratamiento quirúrgico, de la gravedad del proceso clínico (34) y del seguimiento del enfermo (35). La tasa de reingreso es más alta en ancianos, particularmente en el anciano frágil (32) y la insuficiencia cardiaca uno de los problemas médicos con índice de reingreso más alto $(35,37)$.

Paradójicamente a lo que parece esperable, el efecto de la estancia media en ingresos previos, o no tiene influencia (24), o a mayor estancia mayor índice de reingreso (8).

Otros trabajos concluyen que la tasa de reingreso no debe utilizarse como índice de calidad debido a que depende de factores no modificables dependientes de la enfermedad $(6,10)$.

Con el incremento del gasto sanitario debido en parte al envejecimiento de la población, y a pesar de que hace una década se defendía su utilización como estándar de calidad, evitar el mismo no suele ser un objetivo de la política hospitalaria (2). Lo cierto es que como un evento epidemiológico más se ha comenzado a estudiar la prevención del reingreso; así se ha cifrado que $37 \%$ de reingresos dependientes de factores del hospital, $38 \%$ de reingresos por factores clínicos y $21 \%$ de reingresos por factores dependientes del enfermo pueden ser evitados (38). Las medidas que han demostrado valor 
en evitar el reingreso son: la intervención en el plan de alta, la educación del paciente, el seguimiento del mismo (39) y la prevención de iatrogenia durante el ingreso previo (40). En algunos procesos clínicos con alta tasa de reingreso una intervención basada en unidades de enfermería reducen la tasa de reingreso y mejoran la calidad de vida $(41,46)$. También la accesibilidad y rapidez de consulta externa puede influenciar la reducción de reingresos (42), aunque en algunos estudios la accesibilidad a atención primaria tuvo un efecto adverso sobre la tasa de reingreso hospitalario (43) y el control en la consulta externa tras el alta tampoco redundó en reducir dicha tasa (44). Se ha estipulado que el control en consulta externa 2-3 semanas tras el alta hospitalaria es el tiempo ideal para reducir el reingreso (45).

En nuestro país la tasa de reingreso comunicada es muy variable, oscilando entre 16 y $34 \%(48,49)$ apoyándose, en general en las publicaciones españolas, su valor como índice de calidad asistencial hospitalaria (50). La edad (51,57), el sexo masculino $(51,52)$, el estado civil soltero o viudo y la mayor gravedad al ingreso son factores asociados a una tasa alta de reingreso (52). Se ha señalado que la fragmentación de la asistencia, la cultura de la alta especialización y la ausencia de coordinación entre niveles asistenciales son algunas de las causas de los reingresos hospitalarios en nuestro país (54) siendo las enfermedades crónicas tales como enfermedad respiratoria crónica, enfermedad hepática crónica, neoplasias pleuropulmonares (56) y en especial la insuficiencia cardiaca (57) los principales procesos clínicos responsables del reingreso.

En el presente trabajo la tasa de reingreso verdadero es del $26,6 \%$, intermedia entre las tasas publicadas, siendo las enfermedades cardiovasculares crónicas (principalmente la insuficiencia cardiaca) y las respiratorias crónicas las causantes de mayor número de reingresos, hecho concordante con la literatura. La edad de los pacientes totales ya es alta en medicina interna, lo que explica que no haya las diferencias de edad demostradas en otros trabajos. La comorbilidad más importante también corresponde a enfermedades cardiovasculares y la diabetes mellitus.

Los pacientes con reingresos tienen una mayor mortalidad intrahospitalaria que el total de los pacientes. Globalmente, los principales responsables del reingreso son los verdaderos reingresos mientras solo una cuarta parte es debida a nueva patología. A diferencia de otros trabajos, en nuestro estudio son las mujeres las que muestran tasas de reingreso mayores, lo que puede estar relacionado con la mayor expectativa de vida de las mujeres.

Observamos un fenómeno de facilitación en el reingreso hospitalario, de tal manera que una vez que un paciente ingresa en un hospital, este hecho provoca que para las próximas descompensaciones o agravamiento de la enfermedad se produzca más fácilmente el reingreso, en un tiempo más corto (reingreso precoz) y pudiendo recorrer en sucesivos ingresos diferentes áreas del hospital (multingreso).

Parece interesante comentar el hecho de que aquellos pacientes que consultan con atención primaria el reingreso se ve retardado en el tiempo. Al ser un estudio de diseño transversal-retrospectivo no pudimos calcular las veces que se evita el reingreso al consultar con atención primaria. No obstante la frecuencia de consulta con atención primaria es baja.

En nuestro estudio el número de ingresos previos no modifica la estancia hospitalaria, hecho ya señalado en la literatura (53).
Las causas principales de reingreso en estos pacientes son el empeoramiento de la enfermedad crónica, el manejo inadecuado ambulatorio y la iatrogenia-toxicidad. Estos factores pueden ser subsidiarios de prevención. El primero calibrando perfectamente la necesidad de ingreso hospitalario; en este sentido la creación de unidades de hospital de día, unidades de corta estancia u hospitalización a domicilio pueden ayudar a disminuir la tasa de reingreso. El segundo mediante mejoría de la comunicación con atención primaria (disponibilidad de consultas en nivel primario, consulta de problemas clínicos directos entre niveles asistenciales). El tercero, y tal vez es el que mejor identifica la calidad hospitalaria, con la adherencia a guías de práctica clínica para evitar variabilidad clínica. El manejo ambulatorio inadecuado conduce al reingreso del paciente de forma más precoz que las otras causas contempladas. Aunque el tiempo de reingreso en iatrogenia-toxicidad también es más corto, el pequeño número de episodios impide extraer conclusiones estadísticamente válidas.

Aproximadamente $40 \%$ del reingreso fue reingreso temprano, ocurriendo más en aquellos pacientes con mayor número de ingresos previos (facilitación) y asociados a reingreso evitable y a no consultar con atención primaria.

Los pacientes multingresados también muestran un mayor número de ingresos previos (facilitación). En este hecho influyen factores casi siempre derivados del hospital (temporada de ingresos preferentemente en un servicio según demanden en urgencias, interés de la dirección en potenciar un servicio respecto a otro, desvío de pacientes por un determinado especialista de guardia) y menos veces del paciente. Los factores asociados al multingreso son los mismos que los asociados a reingreso verdadero.

Aproximadamente un $20 \%$ del reingreso es hospitalización inadecuada, no cumpliendo criterios de ingreso. Éste también sería un factor subsidiario de prevención; no obstante estos pacientes pasan menos tiempo hospitalizados y presentaron una mortalidad nula.

Observamos que los pacientes procedentes del medio rural tardan más tiempo en acudir al hospital que cuando viven en área urbana. Esto puede explicarse por condicionantes culturales y geográficos, y puede tener su importancia a la hora de planificar atención médica en determinadas patologías donde el factor tiempo es crucial. Los pacientes cuyo médico de atención primaria es especialista en medicina de familia acuden antes al hospital que aquellos con médico de atención primaria de medicina general. Este es un dato de difícil interpretación que probablemente obedezca a más de una causa, desde educacional (formación inicial del especialista en medicina de familia en el hospital), hasta poblacional (diferentes grupos humanos aun dentro de la misma área urbana adscritos a diferentes médicos).

Los principales factores de riesgo de reingreso en estadística multivariante han sido el ser multingreso, el tener un reingreso evitable y cumplir algunas de las causas de reingreso indicadas en la tabla I, no teniendo importancia la dad, el sexo ni el área de residencia ni el tipo de médico de atención primaria.

Opinamos que si bien la tasa bruta de reingreso puede ser un medidor burdo de calidad asistencial, la tasa de reingreso verdadero, la de reingreso temprano y la de reingreso evitable pueden servir como indicador de calidad asistencial de un hospital e incluso de un área de salud, y se ofrecen puntos determinados sobre los que se puede actuar como prevención. 


\section{Bibliografía}

1. Acheson ED, Barr A. Multiple spells of in-patient treatment in a calendar year. British J Preventive and Social Medicine 1965; 19: 182-91.

2. Milne R, Clarke A. Can readmission rates be used as an outcome indicator?, Br Med J 1990; 301: 1139-40.

3. Thomas JW, Holloway JJ. Investigating early readmissions as an indicator for quality od characteristics. Med Care 1991; 29: 377-94.

4. Hofer TP, Hayward RA. Can early readmissions rates accurately detect poor quality hospitals. Med Care 1995; 33: 234-45.

5. Frankl SE, Breeling JL, Goldman L. Preventability of emergent hospital readmission. Am J Med 1991; 90: 667-74.

6. Benbassat J, Taraguin M. Hospital readmissions as a measure of quality of health care: adventages and limitations. Arch Intern Med 2000; 160: 1074-81.

7. Thomas JW. Does risk-adjusted readmission rate provide valid information on hospital quelity? Inquiry 1996; 33: 258-70.

8. Kossovsky MP, Perneger TV, Serasin FP, Bolla F, Borst F, Gaspoz JM Comparison between planned and unplanned readmissions to a department of internal medicine. J Clin Epidemiol 1999; 52: 151-6.

9. Farmer RG, Kay R, Achkar E, Bonecutter TA, Loop FD. Hospital readmissions: a reevaluating of criteria. Clev Clin J Med 1989; 56: 704-8.

10. Clark A. Are readmissions avoidable. Br Med J 1990; 301: 1136-8.

11. Mc Kay MD, Rowe MM, Bernt FM. Disease chronicity and quality of care in hospital readmissions. J Health Qual 1997; 19: 33-7.

12. Weissman JS, Ayanian JZ, Chasan-Taber S, Sherwood MJ, Roth C, Epstein AM. Hospital readmissions and quality of care. Med Care 1999; 37: 490-501.

13. Ashton CM, Kuykendall DH, Johnson ML, Wray NP, Wu L. The association between the quality of impairment care and aerly readmission. Ann Intern med 1995; 122: 415-21.

14. Ashton CM, DelJunco DJ, Souckek J, Wray NP, Mansyur CL. The association between the quality of impairment care and early readmission: a meta-analysis of evidence. Med care 1999; 37: 140-8.

15. Agudo de Blas P, Montoto Otero C, Contreras Murillo E, Casado Messegué $\mathrm{O}$, Conthe Gutiérrez P. Manejo terapéutico inadecuado en pacientes con insuficiencia cardiaca: estudio de los reingresos en un hospital general. An Med Interna (Madrid) 1999; 16: 7-61.

16. Slack R, Bucknall CE. Readmission rates are associated with differences in the process of care in acute asthma. Qual Health Care 1997; 6: 194-8.

17. Philbin EF, DiSalvo TG. Prediction of hospital readmission for heart failure: developing a simple risk score based on administrative data. Rev port Cardiol 1999; 18: 85-6.

18. Ashton CM, Wray NP. A conceptual framework for the study of early readmissions as an indicator of quality of care. Soc Sci Med 1996; 43: 1533-41.

19. Shipton S. Risk factors associated with multiple hospital readmissions. Home Care Provid 1996; 1: 83-5.

20. Williams EI, Fitton F. Factors affecting early unplanned readmisiion of elederly patients to hospital. Br Med J 1988; 297: 784-7.

21. Holloway JJ, Thomas JW. factors influencing readmission risk: implications for quality monitoring. health care Financ Res 1989; 11: 19-32.

22. Anderson GF, Steinberg EP. Hospital readmissions in the Medicare population. N Engl J Med 1984; 311: 1349-53.

23. Gautam P, Modaff C, Brown I, Squair J. Unplanned readmissions of elderly patients. Health Bull (Edim) 1996; 54: 449-57.

24. Chambers M, Clarke A. Measuring readmission rates. Br Med J 1990; 301: 1134-6.

25. Soeken KL, Prescott PA, Heron DG, Creasia J. Predictors of hospital readmission. A meta-analysis. Eval Health Prof 1991; 14: 262-81.

26. Candlish P, Watts P, Readman S, White P, Lowe J. Elderly patients with heart failure: a study of satisfaction with care and quality of life. Int J Qual Health Care 1998; 10: 141-6.

27. Dosaki R, Mizushima Y, Kawasaki A, Hoshino K, Nakagawa K, Ko M. A clinical study on asmathic patients who were readmitted aer a short period of discharge. Kokyu To Jun Kan 1993; 41: 117-36.

28. Marcantonio ER, Mc Kean S, Goldfinger M, Kleefield S, Yurkofski T, Breenan TA. Factors associated with unplanned hospital readmission among patients 65 years of age and older in Medicare. Am J Med 1999; $118 ; 823-32$.

29. Holloway JJ, Medendorp SJ, Bromberg J. Risk factor for early readmission among veterans. Health Ser Res 1990; 25 : 213-17.

30. D'Agostino RS, Jacobson J, Clarkson M, Svenson LG, Williamson DM. Readmission after cardiac operations: prevalence, patterns, predisponing factors. J Cardiovasc Surg 1999; 118: 823-32.
31. Kellog FR, Brickner PN, Conley L, Conroy M. Controlling hospital readmission of elederly persons: risk factor analysis. Home Health Care Searv Q 1991; 12: 5-16.

32. Experton B, Ozminkowski RS, Pearlman DN, Li Z, Thompson S. How does managed care manage the frail elederly? The case of hospital readmissions in fee-forservice versus HMO system. Am J Prev Med 1999; 16: 163-72.

33. Mazei G, Chung F. return hospital visits and hospital readmissions after ambulatory surgery. Ann Surg 1999; 230: 721-7.

34. Vinson JM, Rich MW, Sperry JC, Shah AS, McNamara T. Early readmissions of elederly patients with congestive heart failure. J Am Geriatr Soc 1990; 38: 1290-5.

35. Kumholz HM, Parent HM, Tu N et al. Readmission after hospitalizations for congestive heart failure in medicare beneficiaries. Arch Intern Med 1997; 157: 99-104.

36. Krumholz HM, Chen YT, Wrang Y, Vaccarino V, Radford MJ, Horner $\mathrm{H}$. Predictors of readmission among lederly survivors of admission with heart failure. Am Heart J 2000; 139: 72-7.

37. Oddone EZ, Weinberger M, Horner M et al. Classifying general medicine readmissions. Are they prvenible? J Gen Intern Med 1996; 11: 597 607.

38. Haarsma T, Halfens RJ, Huijer-Abu Saad H. Readmission of older heart failure patients. Prog cardiovasc Nurs 1996; 11: 15-20.

39. Bero La, Lipton HL, Bird JA. Characterization of geriatric drug-related hospital readmissions. Med Care 1991; 29: 989-1003.

40. Rich NW, Beckham V, Wittemberg C, Leven CL, Freedland KE, Carney RM . A multidisciplinary intervention to prevent the readmission of elederlyu patients with congestive heart failure. N Engl J Med 1995; 333: 1190-5.

41. Graham H, Livesly B. Can readmission to a geriatric medical unit be prevented. Lancet 1983; 1: 404-6.

42. Weinberger M, Oddone EZ, Henderson WG. Does increased access to primary care reduce hospital readmissions.lveterans affairs cooperative study group on primary care and hospital readmission. N Engl J Med 1996; 334: 1441-7.

43. Fitzgerald JF, Smith DM, Martín DK, Freedman JA, Katz BP. A care manager intervention to reduce readmissions. Arch Intern Med 1994; 154: $1721-9$

44. Anderson MA, Helms LB, Hanson KS, DeVilde NW. Unplanned hospital readmissions: a home care perspective. Nurs Res 1999; 48: 299 307

45. Andersen HE, Schultz-Larsen K, Kreiner S et al. Can readmission after stroke be prevented? Results of a randomized clinical study. A postdicharge follow-up service stroke survivors. Stroke 2000; 31: 1038-45.

46. Matorras T. El paciente multingresado en medicina interna como tipo especial de readmision hospitalaria. An Med Interna (Madrid) 1990; 7 : 353-7.

47. Sicras Mainar A, Navarro Artieda M. Valoración del reingreso hospitalario en el servicio de medicina interna. Med Clin (Barc) 1993; 101: 732-35.

48. Canosa A, Prat A, Lledó R, Asenjo MA. El reingreso hospitalario como gestión de calidad. Med Clin (Barc) 1997; 108: 317.

49. Peiro S, Librero J, Benages A. Factores asociados al reingreso hospitalario urgente en enfermedades digestivas y hepatobiliares. Med Clin (Barc) 1996; 107: 4-13.

50. Fernández García J, Martínez González MA, García Rodríguez J, Bueno Cabanillas A, Lardelli Claret P, García Martín MA. Factores asociados a la incidencia de reingresos hospitalarios. Med Clin (Barc) 1997 108: 4-8.

51. Castells X, Riu M, Sáez M. Asociación entre la estancia media hospitalaria y la tasa de reingreso prematuro. Med Clin (Barc) 1996; 107: 566 71.

52. Gómez Camacho E, de Villar Conde E, Mayoral Martin L, Grilo Reina A. El reingreso hospitalario en medicina interna. Med Clin (Barc) 1998, 110: 479.

53. Montoro P, Agudo P, Casado O, Contreras E, Conthe P. Reingreso hospitalario por insuficiencia cardiaca en un hospital general. An Med Interna (Madrid) 2000; 17: 71-4.

54. Roige J, Miquel C. El reingreso en un servicio de medicina interna. Med Clin (Barc) 1994; 103: 317.

55. Barba Martín R, Marco Martínez J, Plaza Canteli S et al. Estudio retrospectivo de los reingresos precoces en un servicio de medicina interna. Rev Clin Esp 2000; 200: 252-6. 